\title{
Automated detection and segmentation of thoracic lymph nodes from CT using 3D foveal fully convolutional neural networks
}

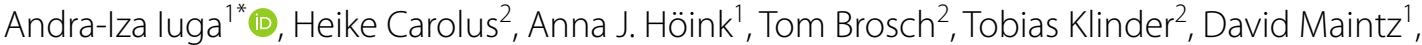 \\ Thorsten Persigehl' ${ }^{1}$, Bettina Baeßler ${ }^{1,3}$ and Michael Püsken ${ }^{1}$
}

\begin{abstract}
Background: In oncology, the correct determination of nodal metastatic disease is essential for patient management, as patient treatment and prognosis are closely linked to the stage of the disease. The aim of the study was to develop a tool for automatic 3D detection and segmentation of lymph nodes (LNs) in computed tomography (CT) scans of the thorax using a fully convolutional neural network based on 3D foveal patches.

Methods: The training dataset was collected from the Computed Tomography Lymph Nodes Collection of the Cancer Imaging Archive, containing 89 contrast-enhanced CT scans of the thorax. A total number of 4275 LNs was segmented semi-automatically by a radiologist, assessing the entire 3D volume of the LNs. Using this data, a fully convolutional neuronal network based on 3D foveal patches was trained with fourfold cross-validation. Testing was performed on an unseen dataset containing 15 contrast-enhanced CT scans of patients who were referred upon suspicion or for staging of bronchial carcinoma.

Results: The algorithm achieved a good overall performance with a total detection rate of $76.9 \%$ for enlarged LNs during fourfold cross-validation in the training dataset with 10.3 false-positives per volume and of $69.9 \%$ in the unseen testing dataset. In the training dataset a better detection rate was observed for enlarged LNs compared to smaller $L N s$, the detection rate for $L N s$ with a short-axis diameter $(S A D) \geq 20 \mathrm{~mm}$ and $S A D$ 5-10 $\mathrm{mm}$ being $91.6 \%$ and $62.2 \%(p<0.001)$, respectively. Best detection rates were obtained for LNs located in Level 4R (83.6\%) and Level 7 (80.4\%).

Conclusions: The proposed 3D deep learning approach achieves an overall good performance in the automatic detection and segmentation of thoracic LNs and shows reasonable generalizability, yielding the potential to facilitate detection during routine clinical work and to enable radiomics research without observer-bias.
\end{abstract}

Keywords: Deep learning, Artificial intelligence, Lymph nodes, Computed tomography, Staging

*Correspondence: andra.iuga@uk-koeln.de

${ }^{1}$ Institute of Diagnostic and Interventional Radiology, Medical Faculty and University Hospital Cologne, University of Cologne, Kerpener Str. 62, 50937 Cologne, Germany

Full list of author information is available at the end of the article

\section{Background}

The correct determination of nodal metastatic disease is imperative for patient management in oncology, since the patients' treatment and prognosis are inherently linked to the stage of disease [1]. For nodal disease staging of solid tumors, unidimensional measurements of lymph node (LN) short-axis diameters (SAD) are routinely performed during tumor staging and re-staging 
imaging examinations and evaluated according to different standardized diagnostic criteria such as the Response Evaluation Criteria in Solid Tumors (RECIST) [2]. For lymphomas, a different set of standardized diagnostic criteria such as the Response Evaluation Criteria in Lymphoma (RECIL) [3] or the Lugano Criteria [4] have been suggested, using bi- instead of unidimensional LN measurements.

Although it is commonly accepted that larger LNs have a higher probability of being malignant as compared to smaller LNs, previous work has shown that enlargement of LNs alone is not the most reliable predictive factor for malignancy with only $62 \%$ sensitivity and specificity being demonstrated for predicting LN metastasis in patients with non-small cell lung cancer when using the proposed $10 \mathrm{~mm}$ cut-off [5]. Consequently, small LNs potentially harboring micrometastases should be taken into consideration for improved diagnostic accuracy during disease staging [6-8]. Unfortunately, no imaging technique (including, e.g., functional techniques such as diffusion-weighted magnetic resonance imaging) so far has been demonstrated to be capable of reliably detecting LN micrometastases [9-11].

Radiomics is a promising novel strategy for predicting LN dignity from images. Radiomic models thereby are built using e.g., machine learning algorithms based on a large set of quantitative features, which are mathematically or statistically derived from medical images [12-14]. For extraction of radiomic features and detection of LN macro- as well as micrometastases, a reliable and correct detection and whole-volume segmentation of small as well as large LNs is needed. Manual or even semi-automated segmentation of LNs is extremely time-consuming and LN detection strongly depends on the radiologist's experience, thus currently hampering the translation of a radiomics-based decision support to clinical routine. Consequently, fully automated approaches are urgently needed for a fast and robust detection and segmentation of LNs.

Recent developments in deep learning (DL) have shown promising results in areas relying on imaging data, especially in radiology $[15,16]$ and cancer imaging $[17$, 18]. While requiring little human input, DL algorithms significantly outperform existing detection and segmentation methods [19], thus offering automated quantification and selection of the most robust features, including a proper 3D assessment of lesions. Moreover, previous work showed that 3D DL architectures were successful in learning high-level tumor appearance features outperforming 2D models [20]. In cancer imaging, the use of artificial intelligence (AI) has shown a great utility not only in the (semi)automatic tumor detection, but also in tumor characterization, and treatment follow-up. In clinical practice, AI has been lately used in digital pathology, in imaging of the brain for the detection of metastasis and in imaging of the chest, for the early detection of breast carcinoma $[17,18]$.

Regarding LNs a wide range of 2D approaches [21, 22] have been proposed so far for detection and segmentation, where LNs were segmented using unidimensional measurements consisting of the determination of the $\mathrm{SAD}$ of the target lesions. However, a unidimensional approach can underestimate the size as well as the growth of LNs, especially when considering enlarged LNs. Consequently, correct segmentation of LNs considering the whole volume of the lesion is of ultimate importance for proper diagnosis and follow-up.

Thus, the aim of the study was to develop a tool for automatic 3D LN detection and segmentation in computed tomography $(\mathrm{CT})$ scans using a fully convolutional neural network based on 3D foveal patches.

\section{Methods}

\section{Description of the training and validation dataset}

For the training and validation dataset, images were obtained from the CT Lymph Nodes Collection of the Cancer Imaging Archive [22]. The dataset can be accessed and downloaded at https://wiki.cancerimagingarchive. net/display/Public/CT+Lymph+Nodes. The dataset was made available to allow for a direct comparison to other detection methods in order to advance the state of the art and to encourage development and improvement of computer-aided detection methods. The dataset contained contrast-enhanced CT images of 90 patients from different scanners with an in-slice resolution between 0.63 and $0.98 \mathrm{~mm}$ and a slice thickness ranging from 1 to $5 \mathrm{~mm}$ (88 CT scans with a slice thickness of 1 or $1.5 \mathrm{~mm}$ and 2 CT scans with a slice thickness of $5 \mathrm{~mm}$ ). To the best of our knowledge, there is no information available regarding patients' disease or further demographic information. The included CT scans showed normal-sized thoracic LNs $(\mathrm{SAD}<10 \mathrm{~mm})$ as well as lymphadenopathy $(\mathrm{SAD} \geq 10 \mathrm{~mm})$. The datasets included also CT scans containing mediastinal bulky disease and bulky axillary lymphadenopathy. In order to allow better comparison to clinical routine with usually heterogeneous datasets, these were not excluded from network training. One case was excluded from our study since it did not contain the complete scan of the thorax.

For this dataset Institutional Review Board approval was not required because it is a publicly available dataset.

\section{Description of the testing dataset}

Further, a second unseen dataset was collected for independent testing. Similar to the training and validation dataset, the testing dataset consisted of 
contrast-enhanced CT scans $(\mathrm{n}=15)$. The patients $(8$ male, 7 female; mean age $68 \pm 16.6$ years) were referred upon suspicion or for staging of bronchial carcinoma from March 2016 to November 2017 (Table 1). All examinations were performed on a 128-slice PET/CT-system (Siemens Biograph mCT Flow 128 Edge, Siemens Medical). Patients were scanned supine in cranio-caudal direction during inspirational breath-hold after intravenous injection of $120 \mathrm{ml}$ contrast medium (Accupaque 350, GE Healthcare) with an injection rate of $2.5 \mathrm{ml} / \mathrm{s}$ and a delay of $60 \mathrm{~s}$. The following scan parameters were used: collimation $128 \times 0.6 \mathrm{~mm}$, rotation time $0.5 \mathrm{~s}$, pitch 0.6 . All axial images were reconstructed with a slice thickness of $2 \mathrm{~mm}$. Similar to the training and validation dataset, the testing dataset included CT scans, that showed both normal-sized thoracic LNs and lymphadenopathy.

Ethical approval was waived due to the retrospective design of the study based on preexisting images (Ethics Committee of the Faculty of Medicine, University of Cologne, reference number 19-1390/ 07.08.2019).

\section{Lymph node segmentation}

\section{Training and validation dataset}

A radiologist (blinded; more than 4 years of experience in thoracic imaging) segmented all LNs of the training and validation dataset with an SAD of at least $5 \mathrm{~mm}$ in the mediastinal, hilar and axillary regions using the semi-automatic 3D Multi-Modal Tumor Tracking tool of a commercially available software platform (IntelliSpace Portal, Version 11.0, Philips Healthcare). In case of unclear LNs or findings CT images were discussed with an experienced radiologist with more than 15 years

Table 1 Demographic details (age and sex) for all patients included in the test dataset

\begin{tabular}{lll}
\hline & Age & Sex \\
\hline 1 & 33 & Female \\
2 & 64 & Female \\
3 & 79 & Male \\
4 & 69 & Female \\
5 & 79 & Male \\
6 & 63 & Male \\
7 & 75 & Male \\
8 & 68 & Male \\
9 & 74 & Female \\
10 & 47 & Male \\
11 & 33 & Female \\
12 & 69 & Female \\
13 & 72 & Male \\
14 & 33 & Female \\
15 & 67 & Male \\
\hline
\end{tabular}

clinical experience and focus in oncological imaging. The training and validation dataset consisted of 4275 LNs, with an average of $48 \mathrm{LNs}$ per patient. When considering the location of the LNs the 4275 LNs included 2272 axillary and 2003 mediastinal/hilar LNs. The LNs had an SAD of 1.3-67.6 mm. A total number of 814 enlarged (SAD $>10 \mathrm{~mm}$ ) LNs was segmented. The segmentation of the LNs took approximately between 45 and 120 min per dataset. LNs with an SAD $<5 \mathrm{~mm}$ that had been mistakenly annotated $(n=690)$ were not included in the evaluation. Figure 1 shows the process of data collection and LN segmentation.

For data evaluation segmented LNs were divided into 3 groups based on their SAD: 5-10 mm (2523 LNs); $10-20 \mathrm{~mm}$ (954 LNs); and > $20 \mathrm{~mm}$ (107 LNs).

Furthermore, based on their localization all segmented LNs were divided into axillary (right, left) and mediastinal (including hilar LNs). The mediastinal LNs were further divided in 11 groups depending on their location corresponding levels (levels 1-11), based on the Mountain-Dresler modification of the American Thoracic Society LN map [23]. The side (right respectively left) was considered for level 1, level 2, level 4, level 10 and level 11.

\section{Testing dataset}

In the testing dataset, a total of $113 \mathrm{LNs}$ were segmented, with an average of $7.5 \mathrm{LNs}$ per patient. The segmented LNs included both axillary and mediastinal/hilar LNs. In this dataset, nevertheless, because of time constraints only LNs with an SAD $>10 \mathrm{~mm}$ were segmented.

\section{Network architecture}

A 3D fully convolutional neural network (u-net) was trained on the training dataset, which obtains as input the original 3D images and the corresponding label masks of the segmented LNs. The output of the network was a probability map, showing the probability of each voxel belonging to a mediastinal or axillary $\mathrm{LN}$. This probability map was assessed with a fixed threshold of 0.4 to obtain the final segmentation result. The threshold value was optimized on the training images to yield the best Dice value over all training samples. Finally, a connected component analysis is applied to obtain the individual predicted LNs.

The segmentation network was trained on the 3D images. The used network architecture, named foveal neural network (f-net) [24] is inspired by the human eye and the distribution of the photoreceptor cells, which have the highest resolution at the fovea centralis. A f-net architecture has been used because this architecture combines information of different resolution levels. On the one hand, LNs were analyzed in high resolution to 


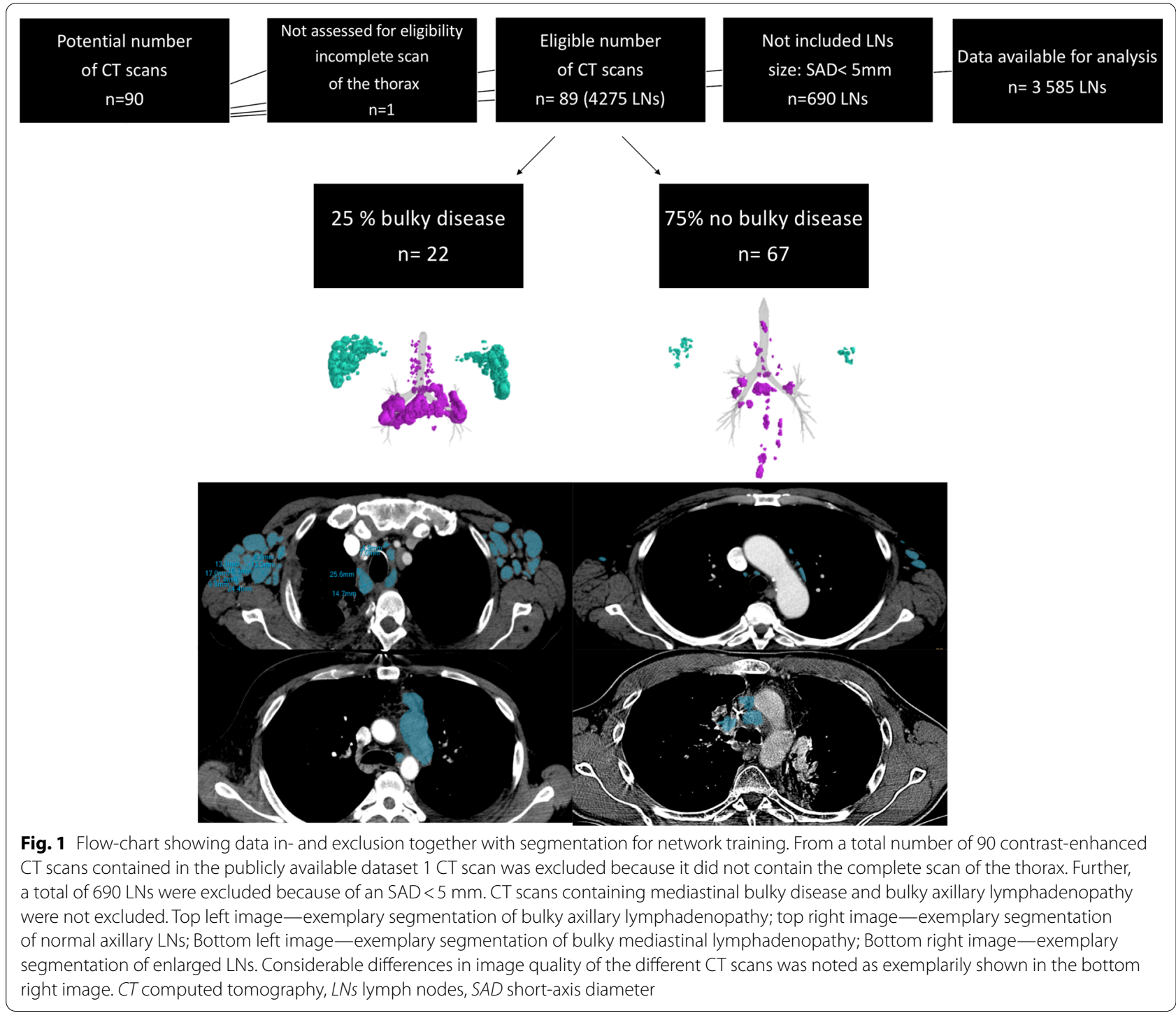

enable feature learning (texture, shape and size). On the other hand, neighboring anatomy was analyzed in low resolution.

As previously mentioned, the network considers image patches at multiple resolution scales in order to arrive at the final prediction, combining local information gained from high resolutions with context from lower resolutions. Unlike u-nets [25], which receive a single scale input image and create the coarser resolution scales by downsampling within the network, f-net directly receives the input as a multiscale pyramid of image patches. Here, an architecture with four resolution levels was used. Accordingly, each input sample to the network consisted of four image patches at the same position but downscaled for the lower resolution levels. The input to each resolution level is processed in a feature extraction pathway. Thus, the number of feature extraction pathways is equivalent to the number of resolution levels in the network. Each feature extraction pathway comprises three successive blocks of valid convolution with a kernel size of 3, batch-normalization, and rectified linear activation function, so called convolutional layer (Conv-L), batch normalization layer (BN-L), and ReLU layer (ReLU-L) (CBR) blocks. The outputs of the feature extraction levels are combined in a feature integration pathway through an additional CBR block followed by upsampling of the lower resolution outputs. Finally, a channel-wise softmax layer is applied to acquire pseudo class probabilities for the LN labels. In addition, f-net was chosen because its architecture requires less memory and runtime compared to u-net [25]. Figure 2 shows an overview of the network architecture. 


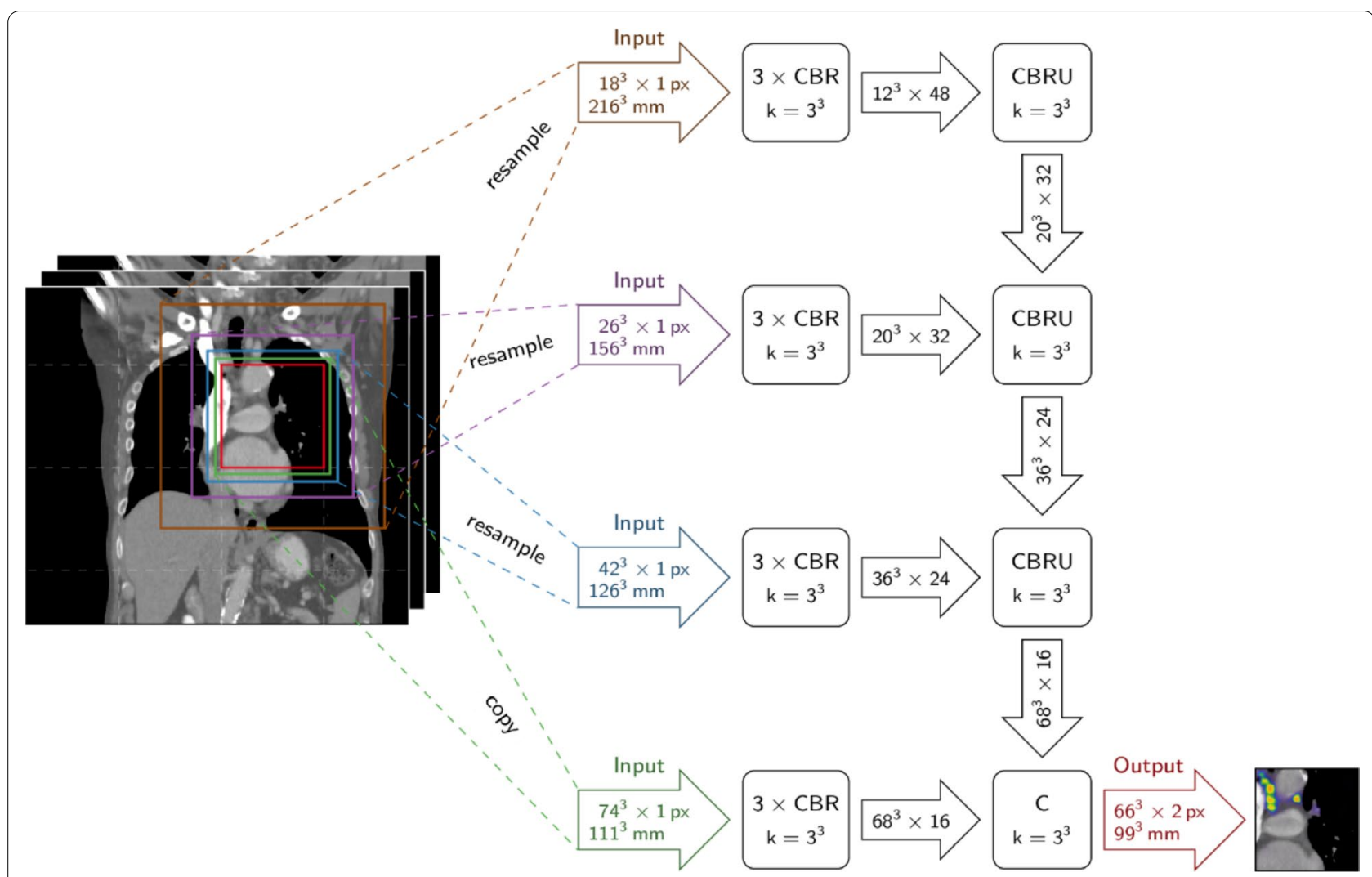

Fig. 2 Sketch of the network architecture. A 3D fully convolutional foveal neural network was trained. The network architecture is inspired by the human eye and the distribution of the photoreceptor cells, which have the highest resolution at the fovea centralis. The network consists of several blocks of convolutional layers, batch normalization and the rectified linear activation function (CBR), which extract features at different resolution levels. CBR blocks are followed by upsampling layers (CBRU) to match the resolution of the other levels

\section{Training and validation setup}

Training was performed using Microsoft Cognitive Toolkit CNTK with a Python interface (Hardware: $2.40 \mathrm{GHz}$ processor with $2 \times$ NVIDIA GTX 1080ti with 11 GB graphics memory). The images were pre-processed by resampling them to a fixed isotropic sampling grid with a spacing of $1.5 \mathrm{~mm}$, this increases the speed of the network training and deployment while preserving sufficient image detail. Used matrix size was standard $(512 \times 512)$ and data pixel size was $1 \mathrm{~mm}$ isotropic.

To enhance the soft-tissue contrast of the LNs, only the gray-value window 750/70 Hounsfield Units (HU) was considered and gray-values outside this range were clipped to the upper or lower limit. This gray-value window was determined automatically on the training data by computing the mean and standard-deviation of all voxels labeled as LN and their direct neighborhood. No further pre-processing was performed.

Training was performed based on patches (hereby, it was ensured that at least $30 \%$ of the patches contain LN voxels), which were drawn randomly from the images. As data augmentation, random scaling and rotation of the patches was applied on-the-fly with a maximal scaling factor of 1.1 and a maximal rotation of $7^{\circ}$. The usage of stronger augmentation with regard to rotation and scaling showed a decline in performance and was therefore abandoned. Individual LNs were not manipulated during data augmentation and therefore the total number of LNs remained unchanged. Test-time augmentation has not been performed.

The cross-entropy function was chosen for the optimization of the network since it showed good performance on many tasks $[12,13,26]$.The network was trained for 1000 epochs with a minibatch size of 8 and the AdaDelta optimizer.

The models were trained using fourfold cross-validation on the training data with the dataset being randomly split into four groups (i.e., training was performed on 3 of the groups while the remaining group was used for validation.). The validation was used to explore performance of the network architecture and training setup with regard to number of resolution levels in the network, optimizer, augmentation and patch sampling strategy. In the following we present the results of the best training 
experiment. A full ablation study is beyond the scope of this paper and will be addressed in future work.

\section{Testing setup}

Finally, the model trained on the complete training dataset was tested on the previously unseen, in-house derived testing dataset.

\section{Evaluation criteria}

The performance of the network is assessed by looking at the individual LNs. For the ground-truth the single nodes are available from the annotation process. For the predicted LNs, a connected component analysis of the predicted segmentation mask is performed.

One performance metric is the detection rate, which is the number of detected LNs divided by the total number of LNs. A LN is thereby counted as detected if there was at least one voxel overlap with the segmentation mask predicted by the network.

The second performance metric is the number of false positives (FP) per volume. Here, a connected component in the predicted segmentation mask without overlap to a ground truth is counted as FP.

This rather loose criterion was chosen instead of stricter measures, e.g., larger overlap thresholds between ground truth and predicted segmentation, as one particular challenge in $\mathrm{LN}$ assessment is that differentiation of individual nodes is often not possible when adjacent nodes merge into clusters due to pathology. Obviously, it can occur that a ground truth segmentation is 'detected' by multiple predicted segmentations and similarly that a predicted segmentation overlaps with multiple groundtruth segmentations. This criterion appears to be current state-of-the-art and has been used in previous work [22].

In addition, the segmentation quality is assessed on a voxel level per image for the detected LNs. To this end, all missed LNs are removed from the ground-truth mask and all FP are removed from the predicted segmentation mask. From the resulting masks Dice, true-positive rate and positive predictive value are computed.

\section{Statistical analysis}

Statistical analysis was performed in the open-source statistics package $\mathrm{R}$ version 3.3.1 for Windows (R: A language and environment for statistical computing, $\mathrm{R}$ Core Team, R Foundation for Statistical Computing. ISBN 3-900051-07-0, 2019, URL http://R-project.org/). After assessing normal distribution of the data, a two-sided unpaired t-test was applied to determine the differences in means of the detection rates considering both size and location of the LNs. Statistical significance was defined as $p \leq 0.05$. To get an impression of the variability of the observed detection rates and to confirm the robustness of the results, bootstrapping analysis was performed (with replacement using 100\% of the sample size with the number of simulations $\mathrm{N}=10.000$ ).

\section{Results}

Calculation of the LN probability maps took about $24 \mathrm{~s}$ per dataset on a graphics processing unit (GPU), while training took 120-180 min.

Bootstrap analysis was performed and confirmed the robustness of the results. The empirical distribution of the detection rate showed a standard deviation of $1.7 \%$.

\section{Network performance: validation dataset Segmentation accuracy}

Overall, a mean Dice value of 0.75 and 0.48 is achieved on the training and validation dataset. True positive rate and positive predictive value account to 0.76 and 0.75 on the training and 0.45 and 0.62 on the validation data. The Dice value for the mediastinal LN accounts to 0.44 and to 0.55 for the axillary $\mathrm{LN}$ with a smaller gap between training and validation, therefore showing less overfitting. More details can be seen in Fig. 3.

\section{Lymph node detection rate according to lymph node size}

The overall detection rate for all LNs with an SAD > $5 \mathrm{~mm}$ using the trained network was $66.5 \%$ with 10.3 FPs per volume on average. Exemplary images of detected and missed LNs compared to the ground truth segmentations are shown in Fig. 4. The highest detection rate could be observed when looking only at LNs with an SAD > $20 \mathrm{~mm}$, while detection rate was only good to moderate when considering smaller LNs $(\mathrm{SAD}>20 \mathrm{~mm}$ vs. SAD 10-20 mm: $91.6 \%$ vs. $75.3 \%, p<0.001$; SAD $>20 \mathrm{~mm}$ vs. SAD 5-10 mm: $91.6 \%$ vs. $62.2 \%, p<0.001$; Fig. 5). Looking only at the subgroup of clinically relevant enlarged LNs (defined by an SAD > $10 \mathrm{~mm}$ ), a total detection rate of $76.9 \%$ was obtained with a significantly higher detection rate for LNs with an SAD $>10 \mathrm{~mm}$ as compared to LNs with an $\mathrm{SAD}<10 \mathrm{~mm}(76.9 \%$ vs. $62.1 \%, p<0.001$; Fig. 5).

\section{Lymph node detection rate according to lymph node location} A better overall detection rate was obtained for the axillary LNs compared to mediastinal LNs (70.0\% vs. $62.3 \%$, $p<0.001$; Fig. 5). A better detection could be observed when looking only at LNs with an SAD > $20 \mathrm{~mm}$, while detection rate was only good to moderate when considering smaller LNs, both for axillary and mediastinal LNs; axillary LNs with an SAD > $20 \mathrm{~mm}$ versus SAD 10-20 mm: $90.5 \%$ versus $74.9 \%, p<0.001$; $\mathrm{SAD}>20 \mathrm{~mm}$ versus SAD 5-10 mm: $90.5 \%$ versus $47.2 \%, p<0.001$; Fig. 5); mediastinal LNs with an $\mathrm{SAD}>20 \mathrm{~mm}$ versus SAD 10-20 mm: $92.3 \%$ versus $75.7 \%, p<0.001$; 


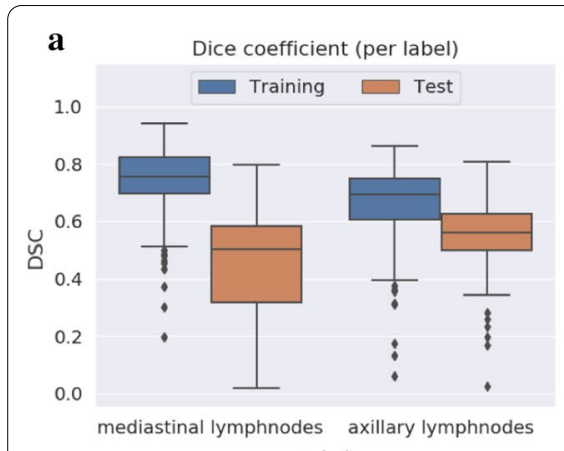

Label

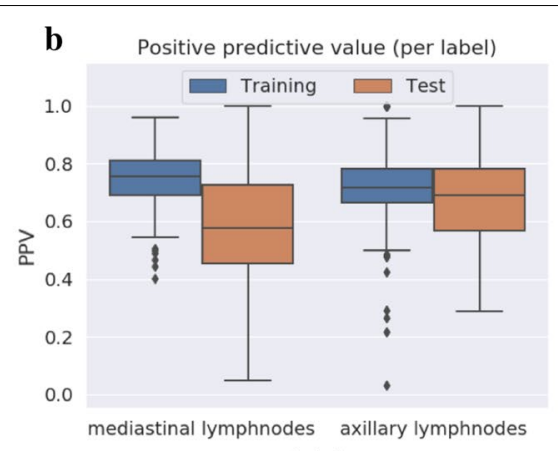

Label

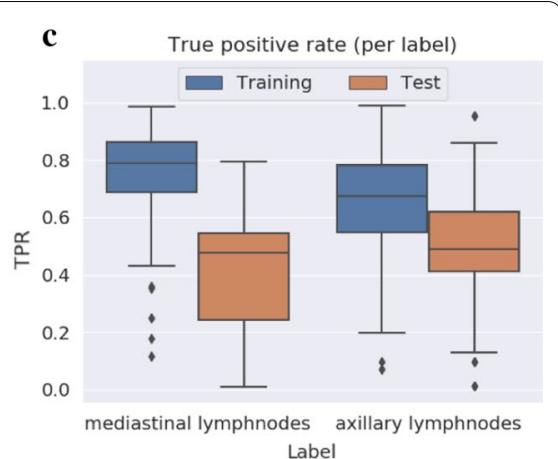

Label

Fig. 3 Overview of dice (a), positive predictive value $(\mathbf{b})$ and true positive rate $(\mathbf{c})$ training and validation data for mediastinal and axillary lymph nodes

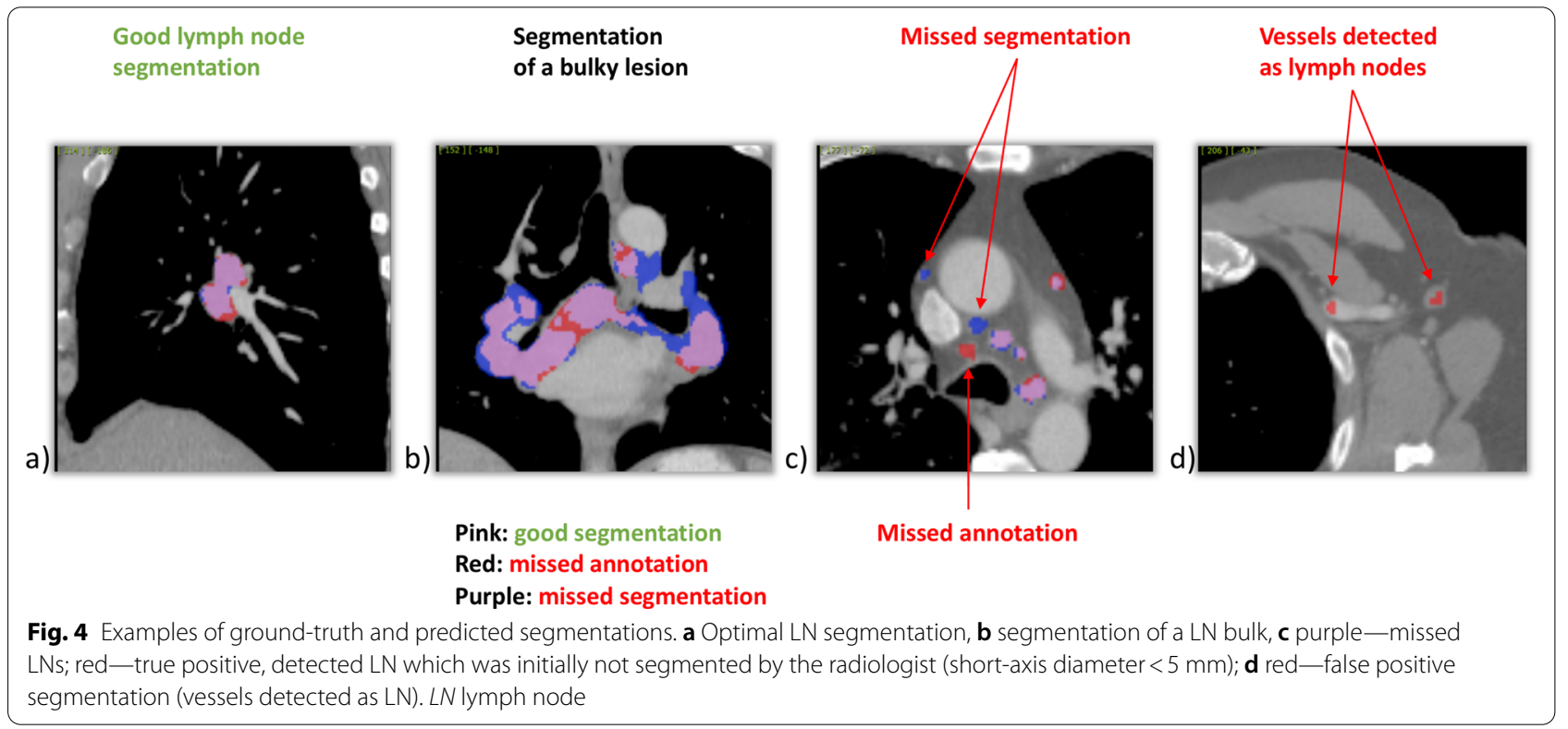

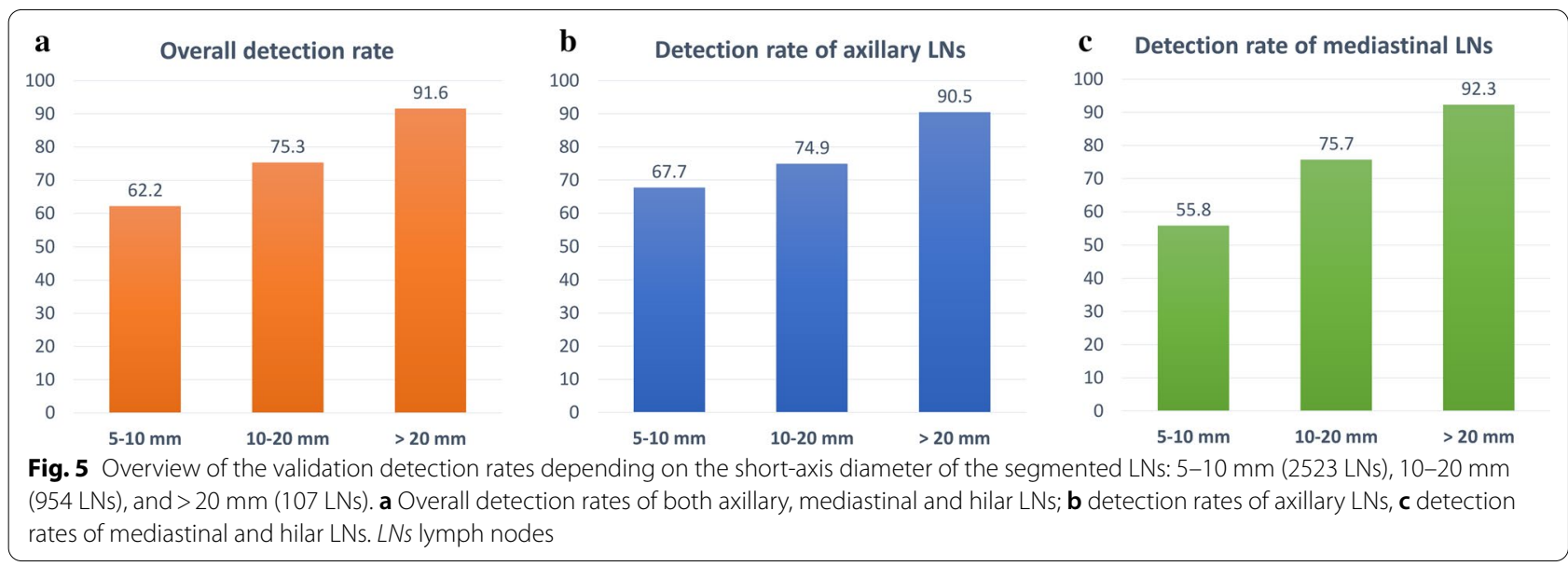


SAD > $20 \mathrm{~mm}$ versus SAD $5-10 \mathrm{~mm}: 92.3 \%$ versus $33.8 \%$, $p<0.001$; Fig. 5). Looking only at the subgroup of clinically relevant enlarged LNs (defined by an $\mathrm{SAD}>10 \mathrm{~mm}$ ), a slightly better detection rate was shown for LNs of the mediastinal region compared to the axillary $(77.8 \%$ vs. $76.0 \%, p<0.05)$.

Based on the labelling of the mediastinal LNs a further analysis was performed to establish detection rates at different levels (Fig. 6). The best detection rates were obtained for LNs located in Level 4R (83.6\%), and Level $7(80.4 \%)$, while the lowest detection rate was recorded for LNs located in Level 8 (25.9\%). A better detection rate was shown for LNs $>10 \mathrm{~mm}$ for all levels. For example, level $2 \mathrm{R}$ (right) showed a detection rate of $96.5 \%$ for LNs $>10 \mathrm{~mm}$ versus $63.5 \%$ for $\mathrm{LNs}<10 \mathrm{~mm}$. For level 7, a total detection rate of $93.3 \%$ was shown for LNs $>10 \mathrm{~mm}$ versus $72.0 \%$ for $\mathrm{LNs}<10 \mathrm{~mm}$. The detection rate was statistically significant different for different levels (Table 2).

\section{Network performance: testing dataset}

On our in-house dataset, which was unseen during training, a detection rate of $69.9 \%$ was achieved for the enlarged LNs (SAD $>10 \mathrm{~mm})$. This result compares well to the $76.9 \%$ achieved on the validation data set. It shows the generalization capabilities of our network which is able to cope with the domain shift when applied to images with a different pathology (bronchial cancer in the testing data, unclear cancer in the training and validation data).

\section{Discussion}

The aim of the study was to develop a 3D DL algorithm for robust LN detection and segmentation in contrastenhanced CT scans of the thorax. The main findings can be summarized as follows: (1) The algorithm achieved a good overall performance with an overall validation detection rate of $70 \%$ for LNs with an SAD over $5 \mathrm{~mm}$. (2) Reasonable generalizability was achieved with a similar detection rate for enlarged LNs $(\mathrm{SAD}>10 \mathrm{~mm})$ in the fourfold cross-validation dataset compared to the unseen testing dataset of $76.9 \%$ and $69.9 \%$, respectively. (3) A better validation detection rate was observed for enlarged LNs compared to smaller LNs (enlarged LNs showed a detection rate of $76.9 \%$; the detection rate for LNs with an SAD $\geq 20 \mathrm{~mm}$ and SAD $0-5 \mathrm{~mm}$ was $91.6 \%$ and $40.8 \%$, respectively). (4). Regarding different LN locations, the best validation detection rates were obtained for LNs located in Level 4R (right mediastinal), Level 7 (mediastinal subcarinal), and Level $10 \mathrm{R}$ (right hilar) of $83.6 \%, 80.4 \%$ and $74.6 \%$, respectively. (5) Segmentation accuracy shows a promising Dice value of 0.48. Segmentation accuracy is superior in the axillary region with less overfitting. This is probably due to the stronger homogeneity of the data compared to the mediastinal LNs.

Although a few DL approaches have been proposed for mediastinal LNs [21, 22, 26], there is still only a very limited number of publications available. A study similar to this work using the same evaluation criteria, employs a 3D u-net with additional organ segmentation

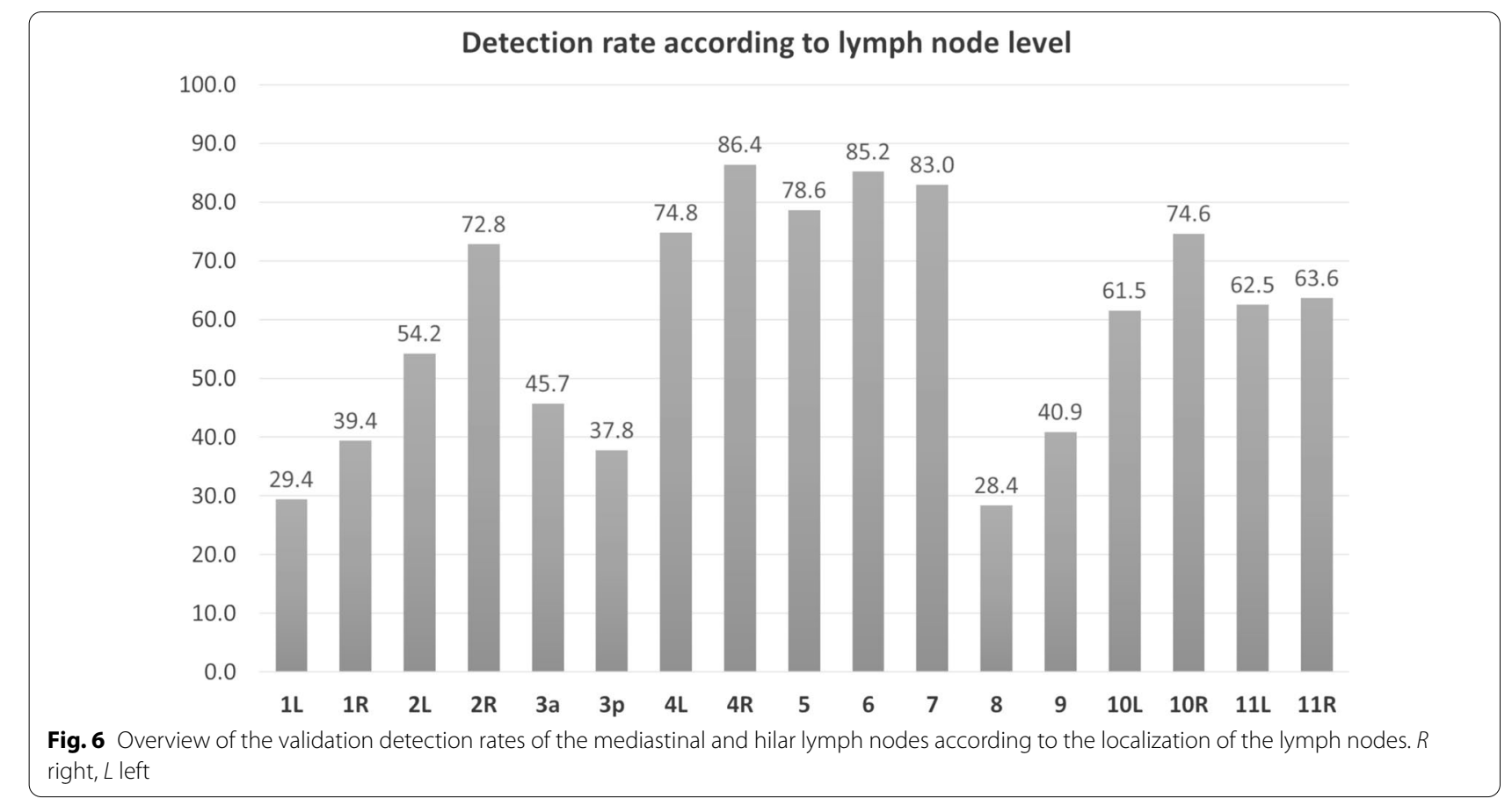




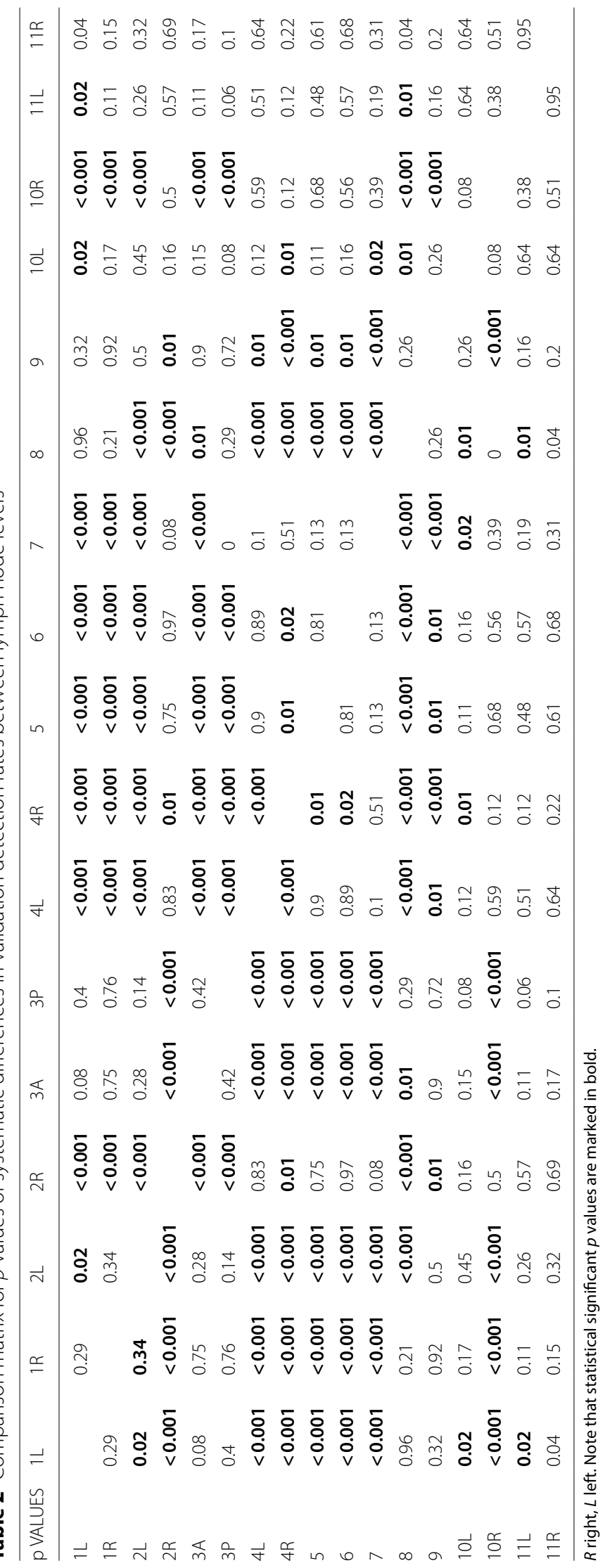


masks as input for mediastinal LN segmentation [26]. A detection rate of $95.5 \%$ is reported on a different dataset including considerably fewer cases, thus impeding comparison to this study. The current approach did not rely on explicit shape modeling nor did it incorporate segmentation of neighboring organs. In addition, both axillary and mediastinal regions were simultaneously addressed, thereby providing a complete assessment of the thoracic region. Moreover, in contrast to other publications a total of $3585 \mathrm{LNs}$ have been used for the training dataset only.

Previous studies using the same public dataset reported detections rates of $78 \%$ [21], 84\% [22] and up to $88 \%$ [27] with 6 FPs per scan. In those studies, only the center of the LN was detected, and a detection was counted as correct if the detected landmark was within a distance of $15 \mathrm{~mm}$ from the ground truth landmark annotation. These detection rates are in good agreement with the validation results of this study while the current approach simultaneously provides a 3D segmentation of the LNs. Therefore, the algorithm can ensure a correct wholevolume segmentation of small as well as large LNs, necessary for the extraction of radiomic features in future approaches. Further, the whole-volume assessment of the network should potentially facilitate future work considering automated determination of total tumor load at diagnosis and in treatment response evaluation.

In contrast to previous studies, where only crossvalidation (sixfold [21, 27] and threefold [22]) was performed, additional testing has been performed on a completely independent previously unseen dataset in addition to the fourfold cross-validation, in order to assess the generalizability of the trained network. Testing showed a similar detection rate compared to the initial fourfold cross-validation dataset, thus achieving a reasonable generalizability and facilitating $\mathrm{LN}$ detection during routine clinical work.

This work considered both axillary and mediastinal LNs using a single convolutional neural network, showing good validation results while addressing two different anatomical regions and therefore offering a complete analysis of the entire thorax with only one network.

Another way to potentially improve the detection rate is by increasing the amount of training data. Multiple, stronger data augmentation strategies, which have not been explored in the present study, have been proposed to improve vision tasks for images $[28,29]$.

CT scans containing bulky axillary or mediastinal lymphadenopathy have not been excluded. Even if the delimitation and segmentation of individual LNs forming the lesions was challenging, consecutively influencing the overall detection $\mathrm{ICH}$ rate negatively, these CT scans ensure a heterogeneous dataset.
The analysis by location showed considerable differences for the different LN levels. For example, for Level 4 LNs a validation detection rate of $85.0 \%$ was achieved for those localized on the right side whereas of only $71.0 \%$ for those on the left side. A possible explanation could be the considerable difference in the number of annotated LNs-262 LNs in level 4R and only $160 \mathrm{LNs}$ in level 4L. A similar difference could be observed for Level $10(75.0 \%$ with 71 annotated LNs for the right side versus 55.0\% with only 29 annotated LNs for the left side). Additionally, worse contrast to surrounding tissue on the left versus right side might be another reason for the differences in detection rates.

Proper LNs classification and labelling is needed in order to develop future approaches in the characterization of malignant LNs, for example when considering Radiomics. Moreover, other features regarding the morphology of the thoracic LNs in addition to size (for example shape or homogeneity) should also be considered in future work. The current work considers just the thoracic LNs. Future work will address the extension to the abdominal region.

The main limitation of this study was the fact that the datasets were segmented by only one radiologist. However, this radiologist was well trained in detection and segmentation of LNs in chest CTs (more than 4 years of experience) and unclear LNs were discussed with an experienced radiologist (more than 15 years of experience). We assumed to have a homogenous dataset of the more than 4.000 manually segmented LNs with optimized inter-rater variability. Nevertheless, in this study the inter-rater effect of independent segmentation datasets for training of the network has not been evaluated. This was beyond the purpose of this study and has to be investigated in a subsequent trail.

Another limitation of the study is the limited number of annotated LNs. Adding more annotations to the training dataset could most probably ensure a better detection rate, especially for the mediastinal LNs located in levels for which the analyzed dataset had just few representatives.

Finally, another limitation of the study is the limited number of data augmentation strategies that has been applied, since multiple and stronger strategies could also potentially improve the detection rate.

\section{Conclusions}

In conclusion, based on extensive and rigorous annotations, the proposed 3D DL approach achieved a good performance in the automatic detection and segmentation especially of enlarged LNs. In contrast to other work, both the axillary and mediastinal regions have been simultaneously addressed and thus a complete 
assessment of the thoracic region is provided. Our approach could be considered for further research regarding quantitative features of LNs to improve and accelerate diagnosis. Extension to other regions should be considered in the future.

\section{Abbreviations}

Al: Artificial intelligence; BN-L: Batch normalization layer; CBR: Convolutional layer (Conv-L), batch normalization layer (BN-L) and ReLU layer (ReLU-L); CBRU: Convolutional layer (Conv-L), batch normalization layer (BN-L) and ReLU layer (ReLU-L), followed by upsampling layers; CNTK: Microsoft Cognitive Toolkit; Conv-L: Convolutional layer; CT: Computed tomography; DL: Deep learning; f-net: Foveal neural network; FP: False positive; GPU: Graphics processing unit; HU: Hounsfield units; LN: Lymph node; RECIL: Response evaluation criteria in lymphoma; RECIST: Response evaluation criteria in solid tumors; ReLU-L: Rectified linear units layer; SAD: Short-axis diameter; u-net: Fully convolutional networks.

\section{Acknowledgements}

The authors would like to thank Martin Balthasar for assistance with the statistical analysis and Jasmin Holz for assistance with data curation.

\section{Authors' contributions}

A-II: Conceptualization, writing - original draft preparation, investigation, validation, visualization, writing — reviewing and editing. HC: conceptualization, writing - original draft preparation, methodology, software, validation, visualization, writing — reviewing and editing. AJH: Conceptualization, supervision. TB: Methodology, software. TK: Conceptualization, supervision, methodology. DM: Conceptualization, supervision. TP: Supervision, writing — reviewing and editing. BB: Supervision, writing —original draft preparation, Writing-reviewing and editing. MP: Conceptualization, supervision, writing-reviewing and editing. All authors read and approved the final manuscript.

\section{Funding}

Open Access funding enabled and organized by Projekt DEAL. This work was partially supported by a Philips Clinical Research Fellowship. The work is part of the SPP initiative of the Deutsche Forschungsgemeinschaft (DFG).

\section{Availability of data and materials}

The training and validation dataset supporting the conclusions of this article is available at https://wiki.cancerimagingarchive.net/display/Public/CT+ Lymph+Nodes or through request to the corresponding author. The testing dataset supporting the conclusions of this article can be accessed through request to the corresponding author.

\section{Declarations}

\section{Ethics approval and consent to participate}

For the training dataset Institutional Review Board approval was not required because it is a publicly available dataset. For the validation dataset ethical approval was waived due to the retrospective design of the study based on preexisting images (ethics committee of the Faculty of Medicine, University of Cologne, reference number 19-1390/ 07.08.2019).

\section{Consent for publication}

Not applicable.

\section{Competing interests}

DM received speaker's honoraria from Philips Healthcare. A-II received institutional research support from Philips Healthcare for research. HC, TB and TK are employees from Philips Research for technical deployment of the Al algorithms. All other authors were independent researchers and guarantee the correctness of the data and results.

\section{Author details}

${ }^{1}$ Institute of Diagnostic and Interventional Radiology, Medical Faculty and University Hospital Cologne, University of Cologne, Kerpener Str. 62,
50937 Cologne, Germany. ${ }^{2}$ Philips Research, Röntgenstraße 24, 22335 Hamburg, Germany. ${ }^{3}$ Institute of Diagnostic and Interventional Radiology, University Hospital Zürich, Zürich, Switzerland.

Received: 4 October 2020 Accepted: 2 April 2021

Published online: 13 April 2021

\section{References}

1. Walker CM, Chung JH, Abbott GF, Little BP, El-Sherief AH, Shepard JAO, et al. Mediastinal lymph node staging: from noninvasive to surgical. AJR. 2012;199:W54-64.

2. Schwartz LH, Bogaerts J, Ford R, Shankar L, Therasse P, Gwyther S, et al. Evaluation of lymph nodes with RECIST 1.1. Eur J Cancer. 2009;45:261-7.

3. Younes A, Hilden P, Coiffier B, Hagenbeek A, Salles G, Wilson W, et al. International Working Group consensus response evaluation criteria in lymphoma (RECIL). Ann Oncol. 2017;2017:1436-47.

4. Cheson BD. Staging and response assessment in lymphomas: the new Lugano classification. Chin Clin Oncol. 2015;4:1-9.

5. De Langen AJ, Raijmakers P, Riphagen I, Paul MA, Hoekstra OS. The size of mediastinal lymph nodes and its relation with metastatic involvement: a meta-analysis. Eur J Cardio-Thorac Chirurgie. 2006;29:26-9.

6. Sloothaak DAM, van der Linden RLA, van de Velde CJH, Bemelman WA, Lips DJ, van der Linden JC, et al. Prognostic implications of occult nodal tumour cells in stage I and II colon cancer: the correlation between micrometastasis and disease recurrence. Eur J Surg Oncol. 2017:43:1456-62.

7. Choi SB, Han HJ, Park P, Kim WB, Song TJ, Choi SY. Systematic review of the clinical significance of lymph node micrometastases of pancreatic adenocarcinoma following surgical resection. Pancreatology. 2017;17:342-9.

8. Leong SPL, Tseng WW. Micrometastatic cancer cells in lymph nodes, bone marrow, and blood: clinical significance and biologic implications. CA Cancer J Clin. 2014;64:195-206.

9. Dappa E, Elger T, Hasenburg A, Düber C, Battista MJ, Hötker AM. The value of advanced MRI techniques in the assessment of cervical cancer: a review. Insights Imaging. 2017;8:471-81.

10. Shen G, Zhou H, Jia Z, Deng H. Diagnostic performance of diffusionweighted MRI for detection of pelvic metastatic lymph nodes in patients with cervical cancer: a systematic review and meta-analysis. Br J Radiol. 2015. https://doi.org/10.1259/bjr.20150063.

11. Otero-García MM, Mesa-Álvarez A, Nikolic O, Blanco-Lobato P, BastaNikolic M, de Llano-Ortega RM, et al. Role of MRI in staging and follow-up of endometrial and cervical cancer: pitfalls and mimickers. Insights Imaging. 2019;10:19.

12. Chen L, Zhou Z, Sher D, Zhang Q, Shah J, Pham NL, et al. Combining many-objective radiomics and 3D convolutional neural network through evidential reasoning to predict lymph node metastasis in head and neck cancer. Phys Med Biol. 2019. https://doi.org/10.1088/1361-6560/ab083a.

13. Spuhler KD, Ding J, Liu C, Sun J, Serrano-Sosa M, Moriarty M, et al. Taskbased assessment of a convolutional neural network for segmenting breast lesions for radiomic analysis. Magn Reson Med. 2019;82:786-95.

14. Ji GW, Zhu FP, Zhang YD, Liu XS, Wu FY, Wang K, et al. A radiomics approach to predict lymph node metastasis and clinical outcome of intrahepatic cholangiocarcinoma. Eur Radiol. 2019;29:3725-35.

15. Litjens G, Kooi T, Bejnordi BE, Setio AAA, Ciompi F, Ghafoorian M, et al. A survey on deep learning in medical image analysis. Med Image Anal. 2017;42:60-88.

16. Ching T, Himmelstein DS, Beaulieu-Jones BK, Kalinin AA, Do BT, Way GP, et al. Opportunities and obstacles for deep learning in biology and medicine. J R Soc Interface. 2018;15:20170387.

17. Bi WL, Hosny A, Schabath MB, Giger ML, Birkbak NJ, Mehrtash A, et al. Artificial intelligence in cancer imaging: clinical challenges and applications. CA Cancer J Clin. 2019;69:127-57.

18. Zhou SK, Greenspan H, Davatzikos C, Duncan JS, van Ginneken B, Madabhushi $A$, et al. A review of deep learning in medical imaging: Image traits, technology trends, case studies with progress highlights, and future promises. 2020; arXiv:2008.09104.

19. Kooi T, Litjens G, van Ginneken B, Gubern-Mérida A, Sánchez Cl, Mann R, et al. Large scale deep learning for computer aided detection of mammographic lesions. Med Image Anal. 2017;35:303-12. 
20. Nie D, Zhang H, Adeli E, Liu L, Shen D. 3D deep learning for multi-modal imaging-guided survival time prediction of brain tumor patients. In: International conference on medical image computing and computerassisted intervention, vol. 9901; 2016. p. 212-20.

21. Seff A, Lu L, Cherry KM, Roth HR, Liu J, Wang S, et al. 2D view aggregation for lymph node detection using a shallow hierarchy of linear classifiers. In: International conference on medical image computing and computerassisted intervention, vol. 17; 2014. p. 544-52.

22. Roth HR, Lu L, Seff A, Cherry KM, Hoffman J, Wang S, et al. A new 2.5D representation for lymph node detection using random sets of deep convolutional neural network observations. In: International conference on medical image computing and computer-assisted intervention; 2014. p. 520-527.

23. Mountain CF, Dresler CM. Regional lymph node classification for lung cancer staging. Chest. 1997;111:1718-23.

24. Brosch T, Saalbach A. Foveal fully convolutional nets for multi-organ segmentation. Medical Imaging. 2018. https://doi.org/10.1117/12.22935 28.

25. Ronneberger O, Fischer P, Brox T. U-net: Convolutional networks for biomedical image segmentation; 2015. arXiv:1505.04597.
26. Oda H, Bhatia KK, Roth HR, Oda M, Kitasaka T, Iwano S, et al. Dense volumetric detection and segmentation of mediastinal lymph nodes in chest CT images. SPIE Med Imaging. 2018. https://doi.org/10.1117/12.2287066.

27. Seff A, Lu L, Barbu A, Roth H, Shin HC, Summers RM. Leveraging midlevel semantic boundary cues for automated lymph node detection. In: International conference on medical image computing and computerassisted intervention. 2015. p. 53-61.

28. Razavian AS, Azizpour H, Sullivan J, Carlsson S. CNN features off-the-shelf: An astounding baseline for recognition. In: IEEE Computer Society conference on computer vision and pattern recognition workshops. 2014. p. $512-9$.

29. Hussain Z, Gimenez F, Yi D, Rubin D. Differential data augmentation techniques for medical imaging classification tasks. In: AMIA annual symposium proceedings. 2017. p. 979-84.

\section{Publisher's Note}

Springer Nature remains neutral with regard to jurisdictional claims in published maps and institutional affiliations.
Ready to submit your research? Choose BMC and benefit from:

- fast, convenient online submission

- thorough peer review by experienced researchers in your field

- rapid publication on acceptance

- support for research data, including large and complex data types

- gold Open Access which fosters wider collaboration and increased citations

- maximum visibility for your research: over 100M website views per year

At BMC, research is always in progress.

Learn more biomedcentral.com/submissions 